ARTICLE

Received 25 Apr 2014 | Accepted 7 Nov 2014 | Published 12 Dec $2014 \quad$ DOl: 10.1038/ncomms6785

\title{
Time-resolved compression of a capsule with a cone to high density for fast-ignition laser fusion
}

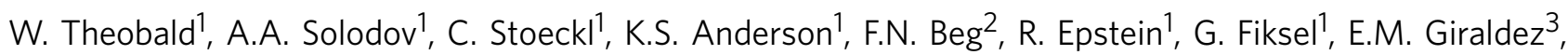

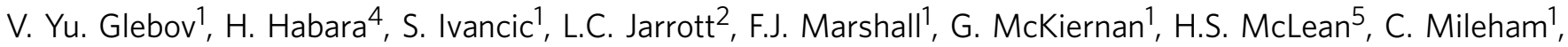
P.M. Nilson ${ }^{1}$, P.K. Patel ${ }^{5}$, F. Pérez ${ }^{5}$, T.C. Sangster ${ }^{1}$, J.J. Santos ${ }^{6}$, H. Sawada ${ }^{7}$, A. Shvydky ${ }^{1}$, R.B. Stephens ${ }^{3}$ \& M.S. Wei ${ }^{3}$

The advent of high-intensity lasers enables us to recreate and study the behaviour of matter under the extreme densities and pressures that exist in many astrophysical objects. It may also enable us to develop a power source based on laser-driven nuclear fusion. Achieving such conditions usually requires a target that is highly uniform and spherically symmetric. Here we show that it is possible to generate high densities in a so-called fast-ignition target that consists of a thin shell whose spherical symmetry is interrupted by the inclusion of a metal cone. Using picosecond-time-resolved X-ray radiography, we show that we can achieve areal densities in excess of $300 \mathrm{mg} \mathrm{cm}^{-2}$ with a nanosecond-duration compression pulsethe highest areal density ever reported for a cone-in-shell target. Such densities are high enough to stop $\mathrm{MeV}$ electrons, which is necessary for igniting the fuel with a subsequent picosecond pulse focused into the resulting plasma.

\footnotetext{
${ }^{1}$ Laboratory for Laser Energetics and Fusion Science Center for Extreme States of Matter, University of Rochester, 250 East River Road, Rochester, New York 14623-1299, USA. ${ }^{2}$ Department of Mechanical and Aerospace Engineering, University of California-San Diego, La Jolla, California 92093, USA. ${ }^{3}$ General Atomics, San Diego, California 92186, USA. ${ }^{4}$ Graduate School of Engineering, Osaka University, Suita, Osaka 565-0871, Japan. ${ }^{5}$ Lawrence Livermore National Laboratory, Livermore, California 94550, USA. ${ }^{6}$ University of Bordeaux, CEA, CNRS, CELIA (Centre Lasers Intenses et Applications), UMR 5107, F-33405, Talence, France. ${ }^{7}$ Department of Physics, University of Reno, Reno, Nevada 89557, USA. Correspondence and requests for materials should be addressed to W.T. (email: wthe@lle.rochester.edu).
} 
$\mathrm{O}$ ver the last four decades, a tremendous effort has been devoted to studying the physics of high-density matter by compressing spherical shells with powerful laser beams $^{1,2}$. Achieving high compression with pressures $\sim 10^{16} \mathrm{~Pa}$ is vital for inertial confinement fusion ${ }^{3}$, where a few milligrams of frozen deuterium (D) and tritium (T) fuel are compressed by laser light ablation (direct drive) or by X-ray ablation (indirect drive) to such high temperatures and densities that ignition is reached and a thermonuclear burn wave spreads throughout the shell. Laboratory compression experiments reach similar pressures that exist inside astrophysical objects, providing an important means for studying those material states ${ }^{4,5}$. Pressures of $\sim 10^{16} \mathrm{~Pa}$ prevail in the sun's core, whereas they are $\sim 100 \times$ lower in the core of giant planets. An important step towards ignition has been recently demonstrated by measuring fusion energy that exceeds the energy coupled in the fuel in an inertial confinement fusion implosion ${ }^{6}$. So far, ignition has not been reached despite code predictions. Besides the conventional 'hotspot' approach, which triggers ignition in the centre of a rapidly converging shell, alternative approaches such as fast ignition (FI) ${ }^{7}$ and shock ignition ${ }^{8}$, which separate the compression and ignition phases of the implosion, have been proposed. Ignition is achieved from highly localized heating: on the side of the high-density fuel using a separate ultrahigh-intensity laser (FI) or in the centre of the compressed shell by a converging shock wave (shock ignition). These concepts are attractive because higher gains might be achievable than with central hot-spot ignition ${ }^{9}$.

Early experiments studying the FI concept ${ }^{0,11}$ reported a coupling efficiency of $15-30 \%$ of the short-pulse laser energy into the compressed plasma. Those experiments were limited by several factors, including insufficient drive-laser energy $(2.5 \mathrm{~kJ})$, no pulse-shaping capability and a longer drive-laser wavelength (532 nm instead of $351 \mathrm{~nm}$ ) - unfavourable factors for achieving high compression. In addition, the low number (nine) of overlapping drive beams resulted in a large illumination nonuniformity that affected the symmetry and possibly the hydrodynamic stability of the implosion. Based on our experiments and simulations, we conclude that the FI experiments ${ }^{10,11}$ were merely qualitative and were not guided by sufficient simulation capability to adequately describe this complicated radiation-hydrodynamic problem. It therefore seems questionable whether sufficient compression was achieved to stop $\mathrm{MeV}$ electrons and surprising that such high coupling efficiencies were reported ${ }^{10,11}$ (efficiencies that could not be reproduced in experiments on $\mathrm{OMEGA}^{12}$ ). Follow-up experiments at the Institute for Laser Engineering confirm an insufficient compression, being $\sim 10 \times$ lower than was previously estimated, and a much-lower $(\sim 1.6 \%)$ short-pulse beam energy coupling ${ }^{13}$. The poor performance of the implosions at the Institute for Laser Engineering might be the result of several factors including a high adiabat $(\alpha>\sim 6)$ in the shell ( $\alpha$ is defined as the ratio of the plasma pressure in the shell to the Fermi pressure of a degenerate electron gas ${ }^{14}$ ), an improperly shaped adiabat profile, breakup of the shell because of hydrodynamic instabilities, or a combination of these factors.

A low adiabat $(\alpha=\sim 1$ to $\sim 2)$ is a prerequisite for high-target compression and is achieved by shaping the laser pulse in such a way that the inner portion of the shell, which is not ablated, remains on a low $\alpha$, whereas the laser raises the $\alpha$ in the ablated outer material in order to reduce the growth of hydrodynamic instabilities ${ }^{15}$. Imploding massive shells with a low implosion velocity $\left(\leq 2 \times 10^{7} \mathrm{~cm} \mathrm{~s}^{-1}\right)$ and a shaped low- $\alpha$ profile substantiates a viable path for achieving highly compressed cores for $\mathrm{FI}^{16}$.

In the experiments reported here, plastic shells with an embedded hollow cone made out of high- $Z$ material were a

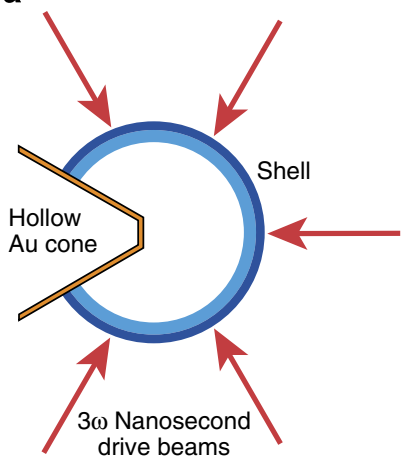

b

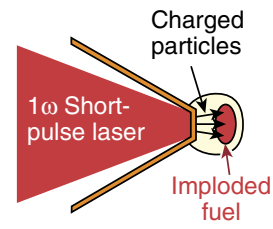

Figure 1 | Schematic of a direct-drive cone-in-shell experiment.

(a) A nanosecond laser implodes a shell consisting of an ablator and a fuel layer around a hollow cone that provides a clear path for a short-pulse laser. (b) The short-pulse laser produces charged particles that ignite the compressed fuel.

imploded with good laser illumination uniformity using the adiabatic shaping technique ${ }^{15}$. Figure 1 shows a schematic of a direct-drive cone-in-shell experiment. The purpose of the cone is to provide a plasma-free path for the short-pulse ignition laser to propagate as close as possible to the dense fuel. One complication is that the cone breaks the spherical symmetry and requires multidimensional simulation capability to accurately model both the flow of the material and the interaction between radiation and matter.

Important quantities that characterize the fuel assembly are the mass-density distribution $\rho(r)$ and the areal density $\rho R$, which is given by the integral of $\rho$ over the spatial coordinate from the shell's centre to infinity, $\int_{0}^{\infty} \rho \mathrm{d} r$. Measurements of $\rho R$ and $\rho$ provide a method for comparing the actual and predicted implosion performances. $\rho R$ and $\rho$ can be obtained experimentally by X-ray radiography ${ }^{17}$. This technique has been used on OMEGA to study the implosion dynamics of deuterium gas-filled plastic shells without a cone using an $\sim 1$-ns-laser-driven, $\sim 5-\mathrm{keV}$ broadband backlighter and an X-ray framing camera with an $\sim 40$-ps integration time ${ }^{18}$. A mass-density distribution $\rho(r)$ and a $\rho R$ of up to $\sim 60 \mathrm{mg} \mathrm{cm}^{-2}$ were measured at various times outside of peak compression ${ }^{18}$. Previous experiments with cone-in-shell targets in indirect ${ }^{19}$ and direct drive ${ }^{20,21}$ investigated high-adiabat $(\alpha=6)$ implosions with simple square pulses, which lacked quantitative comparison to simulations.

Here we demonstrate with picosecond, time-resolved radiographic measurements of the hydrodynamic evolution of cone-inshell implosions that the assembled plasma is dense enough to stop $\mathrm{MeV}$ electrons at a time when the cone tip has not been breached by the implosion-an important requirement for FI. This is only possible because of the unique experimental capability of the Omega Laser Facility and a significant improvement in multidimensional simulations. The excellent agreement between experiment and simulation obtained here provides a benchmark for the code calculations that will be used to further improve implosion performance.

\section{Results}

Experimental setup. The current experiment used thicker $(42 \mu \mathrm{m}$ compared with $24 \mu \mathrm{m}$ ) plastic shells with a cone and a lower gas pressure ( $\sim 0.8 \mathrm{~atm}$ of air compared with $15 \mathrm{~atm}$ of $\mathrm{D}_{2}$ gas) imploded by a shaped laser pulse to minimize X-ray self-emission. The previous experiments with the thinner shell and the higher gas pressure were limited by a strong X-ray self-emission 
a

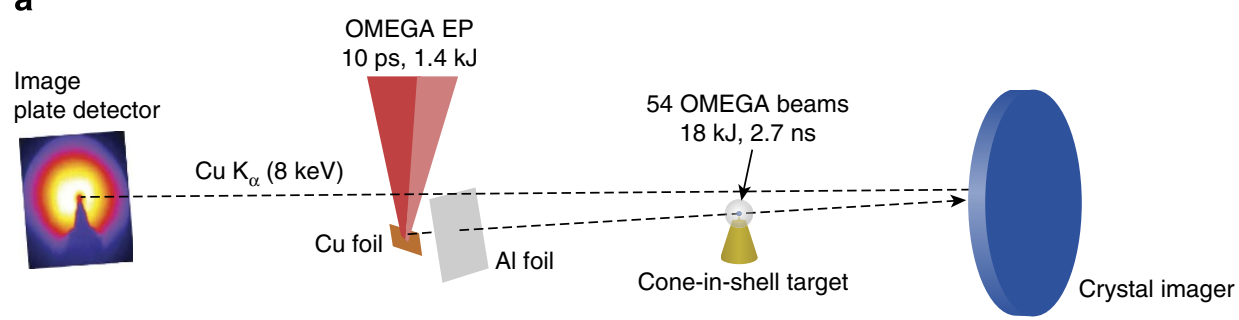

b

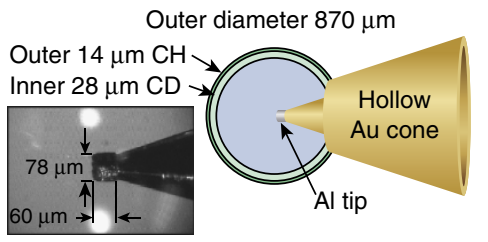

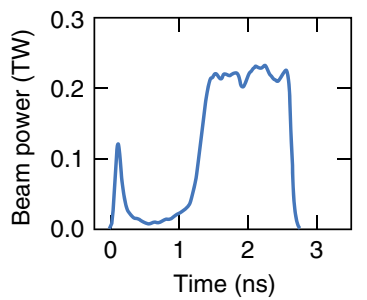

d

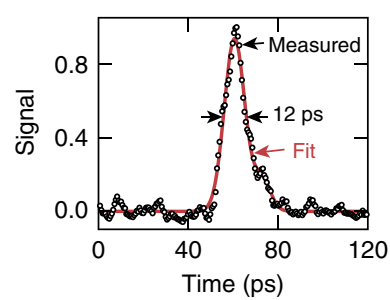

Figure 2 | Experimental setup. (a) Schematic of the setup of the backlighter experiment with a Cu foil irradiated by the OMEGA EP short-pulse beam and using 54 OMEGA beams to implode a cone-in-shell target. A 50- $\mu$ m-thick Al foil was located $2 \mathrm{~mm}$ from the Cu foil to shield it from plasma and X-ray radiation from the implosion. A raw image of the fuel assembly is shown in the image plane. The distances are not to scale. (b) Illustration of the cone-inshell target. (c) Laser pulse shape to implode the capsule. (d) Time-resolved $K_{\alpha} \mathrm{X}$-ray emission from a Cu foil target irradiated with an $\sim 1-\mathrm{kJ}$, 10-ps pulse.

from the hot core that prevented a measurement at peak compression ${ }^{18}$. Here a short-pulse laser-driven X-ray source with shorter emission time and a higher photon energy combined with a narrow-bandwidth $\left(\Delta E / E=1.2 \times 10^{-3}\right)$ crystal imager provided the necessary tool to study the fuel assembly in unprecedented detail. The mass-absorption coefficient $\mu$ of the compressed material is a function of the photon energy, and measuring it with a spectrally pure photon source significantly decreases the uncertainty in the inference of $\rho$ and $\rho R$. Figure 2a shows the experimental setup. The cone-in-shell target (Fig. 2b) consisted of a hollow gold cone mounted inside a plastic shell. A small aluminium cylindrical tip was mounted on the end of the cone. The purpose of the $60-\mu \mathrm{m}$ thick $\mathrm{Al}$ tip was to delay the shock breakout compared with a previous design with a $15-\mu \mathrm{m}$ $\mathrm{Au}$ tip ${ }^{12}$. There is a trade-off between possessing sufficient tip material to delay the breakout and a good electron coupling into the core because more material might affect the electron transport and increases the standoff distance from source to core. The new design does not worsen the electron transport (see Discussion). After imploding the shell with 54 OMEGA ultraviolet beams ${ }^{1}$ with an energy of $\sim 18 \mathrm{~kJ}$ and the drive pulse shown in Fig. $2 \mathrm{c}$, the infrared (1053 nm wavelength), 1.4-kJ, 10-ps OMEGA EP short-pulse laser ${ }^{22}$ irradiated a thin $\mathrm{Cu}$ foil. The OMEGA EP laser was defocused to an $\sim 200-\mu \mathrm{m}$ spot that provided an intensity of $\sim 5 \times 10^{17} \mathrm{~W} \mathrm{~cm}^{-2}$ and generated fast electrons with a kinetic energy in the range of several $100 \mathrm{keV}$ to $\sim \mathrm{MeV}$ (ref. 23). Strong electrostatic sheath fields at the target boundary retain most of the fast electrons in the $\sim 1-\mathrm{mm}$ foil. The electrons recirculate and generate $K_{\alpha}$ radiation, providing a relatively uniform $\mathrm{Cu} K_{\alpha}$ area backlighter source. A spherical Bragg crystal imager ${ }^{24}$ tuned to the $\mathrm{Cu} K_{\alpha 1}$ line $(8.048 \mathrm{keV})$ was located on the opposite side of the target and imaged the implosion onto an image plate detector with a magnification of 14.7. The imaging system efficiently rejects unwanted background and X-ray self-emission from the implosion. The technique also benefits from a higher probing photon energy because the plasma self-emission scales with $\exp (-h v / k T)$, where $h v$ is the photon energy and $k T$ is the plasma temperature. In shots without a backlighter, the background at $8 \mathrm{keV}$ caused by self-emission from the implosion was measured to be $\sim 40 \times$ weaker than the signal

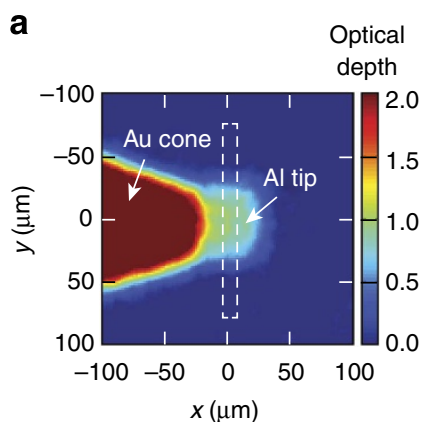

b

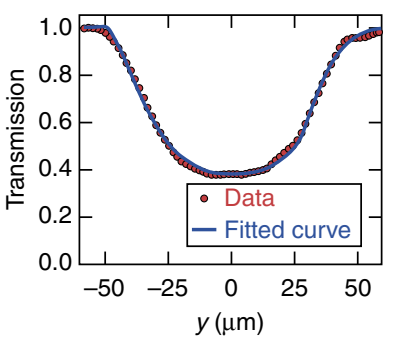

Figure 3 | Radiograph of undriven target. (a) Flash radiography image of an undriven cone-in-shell target and (b) vertical lineout through the centre of the Al tip calculated from the area marked by the dashed rectangle. The measured transmission is well fitted using the 8.048-keV X-ray transmission through a cold aluminium disk.

of the $K_{\alpha}$ backlighter. Another important parameter for this technique is the emission time of the backlighter, which determines the time resolution, because a time-integrating detector was used. Figure $2 \mathrm{~d}$ shows a time-resolved measurement of the $K_{\alpha}$ flash $^{23}$ by coupling an ultrafast X-ray streak camera to the Bragg crystal imager. A $K_{\alpha}$ emission time of 12 ps was quantified, which is short enough to prevent any spatial blurring from the hydrodynamic motion.

Radiographic images. First, an image of an undriven target was taken (see Fig. 3a), where the Al tip is clearly visible and less opaque than the gold cone. This shot provided an independent measurement of the magnification of $14.8 \pm 0.1$ and a measure of the spatial resolution of $22 \pm 3 \mu \mathrm{m}$. The backlighter was then used to image imploded targets. The time delay between the implosion laser and short-pulse laser was varied on a shot-to-shot basis to probe the fuel assembly at various times (see Fig. 4a-e). The false colour scheme represents the measured optical depth given by $\ln \left(I_{0} / I\right)$, where $I$ is the measured transmitted signal and $I_{0}$ is the 

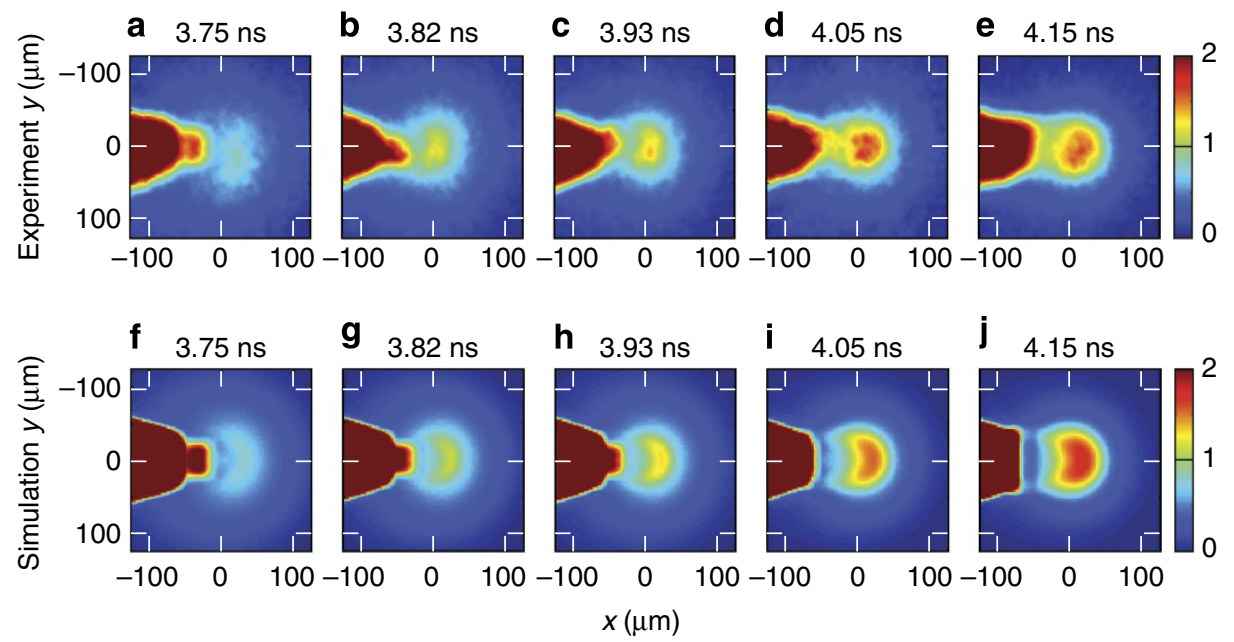

Figure 4 | Radiographic images of implosion. (a-e) Measured images of cone-in-shell targets at various stages of the implosion. The probing time was measured in situ with \pm 0.05 -ns accuracy at full energy by operating the neutron temporal diagnostic ${ }^{38}$ in hard $X$-ray mode. The probing time is with respect to the start of the ultraviolet drive-laser pulse. (f-j) Simulated radiographs from 2D radiation-hydrodynamic simulations. The false colour scheme indicates the optical depth.

measured backlighter intensity. The frame in Fig. 4a shows the implosion at $3.75 \mathrm{~ns}$ after the start of the drive pulse. Time zero is defined as the time when $2 \%$ of the peak power from the drive laser pulse is reached. The fuel starts to assemble in front of the tip and the Al tip is compressed to a higher density, which can be seen when comparing the measured optical depth in the Al tip to that of the undriven target. At later times, the Al tip was more deformed and eventually completely destroyed as the fuel assembly reached higher density. Peak compression was reached at the time of the frame in Fig. $4 \mathrm{~d}$ at $T_{\text {peak }}=4.05 \mathrm{~ns}$, whereas the last frame was recorded after peak compression at $4.15 \mathrm{~ns}$. Figure $4 \mathrm{f}-\mathrm{j}$ displays calculated backlighter images for those shots. The simulations were performed with the two-dimensional (2D) radiation-hydrodynamic code $D R A C O^{25}$ in cylindrical geometry and were post-processed with the code Spect $3 D^{26}$. The simulation capability was significantly improved compared with the results reported in ref. 12. The radiation transport has been included, the Eulerian scheme improved, and nonlocal thermal electron transport ${ }^{27}$ and cross-beam energy transfer ${ }^{28}$ accounted for.

Shock breakout. The breakout time of the shock driven through the cone tip was measured independently, similar to experiments that were described in detail in refs 12,21. The shots were performed by firing only the OMEGA beams and switching off the OMEGA EP beam. The compressing shell pushed a jet of plasma material towards the cone tip and created a shock wave through the cone wall. When the shock wave reached the inner cone surface, it generated optical emission, which was measured temporally and spatially resolved. The measured average breakout time from three implosions was $T_{\text {shock }}=3.85 \pm 0.04 \mathrm{~ns}$, which agrees well with the predicted value of $3.84 \mathrm{~ns}$.

Density profiles. We inferred the time-resolved radial density distributions and time-resolved areal densities from the measured optical-depth distributions under the assumption of cylindrical symmetry of the plasma along the cone axis. Vertical lineout profiles of the optical depth $\tau(y)$ (see Fig. 5) taken through the dense core show maximum values of up to 1.65 and a full-width at half-maximum as small as $\sim 80 \mu \mathrm{m}$. Experimental and simulated $\rho(r)$ are compared in Fig. $6 \mathrm{a}-\mathrm{e}$ at various times. The $\rho(r)$

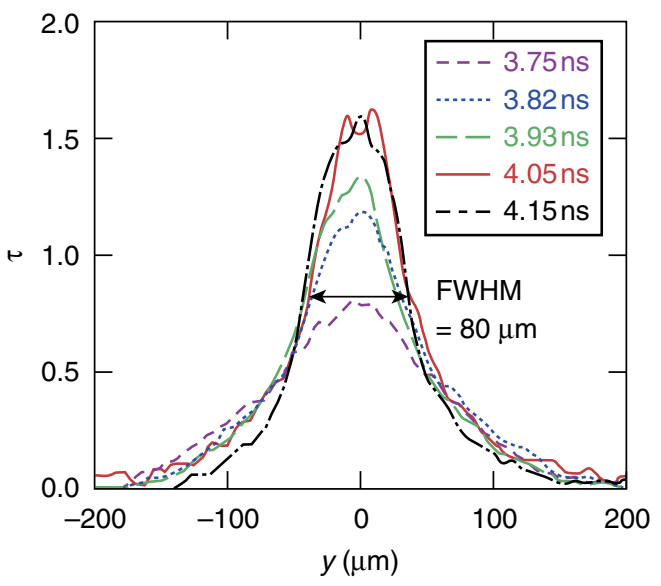

Figure 5 | Optical-depth profiles. Time-resolved optical-depth lineouts through the dense core of compressed plastic measured at a photon energy of $8.048 \mathrm{keV}$. Profiles are taken perpendicular to the cone axis. FWHM, full-width at half-maximum.

profiles match for radii larger than $50 \mu \mathrm{m}$ but show slight differences in the dense region.

Areal density. The inferred $\rho(r)$ is integrated to obtain $\rho R$, which is compared with the predicted value (Fig. 7). The measured areal densities agree with the simulations to better than $15 \%$. The laser compressed the plasma to high areal density $\left(\sim 300 \mathrm{mg} \mathrm{cm}^{-2}\right)$ at a distance of $\sim 50 \mu \mathrm{m}$ in front of the cone tip.

\section{Discussion}

The simulations compare well with the measured radiographic images, revealing similar structure and size of the imploded plastic material. The simulated optical densities agree to better than $10 \%$ with the measured optical densities. Slight differences are observed in the shape of the high-density region, which might be caused by three-dimensional effects that were not taken into account in the simulations. For late times, some differences are observed in the region between the destroyed Al tip and the dense 

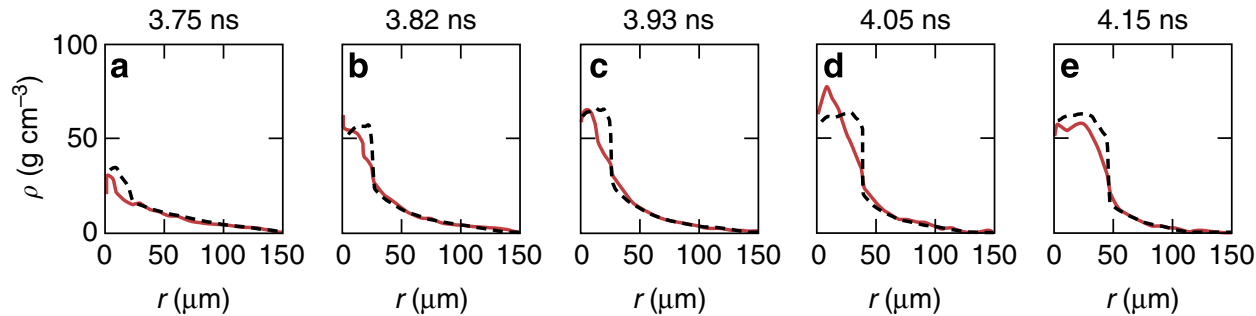

Figure 6 | Density profiles. Measured (solid) and simulated (dashed) density profiles perpendicular to the cone axis for various times (a-e).

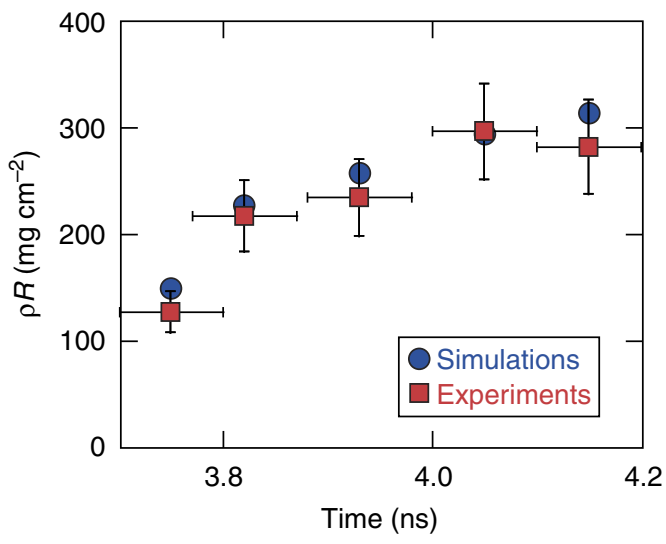

Figure 7 | Areal density. Measured (squares) and simulated (dots) areal density perpendicular to the cone axis for various times. $\rho R$ is determined from the core centre to infinity. Error bars in time are based on the measurement accuracy of the timing diagnostics ${ }^{38}$. The $\rho R$ error bars are based on a $14 \%$ uncertainty in the inferred $\rho R$, which is determined from the errors in the measured optical depth $\tau(12 \%)$ and the mass absorption coefficient $\mu$ (7\%). The $\tau$-error includes contributions from uncertainties in background subtraction, photon statistics and the reconstruction of the 2-D backlighter intensity distribution. The $\mu$-error is inferred based on an estimated $10 \%$ temperature uncertainty.

plasma core (see Fig. 4). Although the simulations show a distinctive gap, the gap is less pronounced in the measurements, which could be a result of turbulent plasma behaviour and mixing of ablated $\mathrm{Al}$ material with the plastic. No significant mixing of $\mathrm{Al}$ and plastic material is noticed in the $2 \mathrm{D}$ simulations but could be stronger in reality. Slight shot-to-shot differences in the Al-tip deformation do not affect the overall performance of the implosion.

The present target is more resilient against the strong shock from the implosion than a previous design with a gold-only cone ${ }^{12}$. Those experiments measured the breakout time for various gold-cone tips with thicknesses from 5 to $15 \mu \mathrm{m}$ and demonstrated a later breakout for thicker tips. For a $15-\mu \mathrm{m}$ thickness, the breakout time was $3.76 \mathrm{~ns}$. Therefore, the target with the $60-\mu \mathrm{m}$-thick $\mathrm{Al}$ tip improved the margin for the arrival of the short-pulse laser by $\sim 90 \mathrm{ps,} \mathrm{bringing} \mathrm{the} \rho R$ at $3.85 \mathrm{~ns}$ to $\sim 200 \mathrm{mg} \mathrm{cm}^{-2}$ (Fig. 7), which is $\sim 70 \%$ of the peak $\rho R$. The Altip target does not worsen the transport of fast electrons through the tip as the areal density of the $60-\mu \mathrm{m} \mathrm{Al}+2-\mu \mathrm{m} \mathrm{Au}$ tip is $20 \mathrm{mg} \mathrm{cm}^{-2}$, whereas the areal density of the $15-\mu \mathrm{m}$-thick Au tip is $29 \mathrm{mg} \mathrm{cm}^{-2}$. The continuous-slowing-down-approximation ranges of fast electrons in $\mathrm{Al}$ and $\mathrm{Au}$ are comparable: $555 \mathrm{mg} \mathrm{cm}^{-2}$ and $776 \mathrm{mg} \mathrm{cm}^{-2}$ (ref. 29) for $1-\mathrm{MeV}$ electrons, respectively. Slightly less areal density is required in $\mathrm{Al}$ to completely stop a $1-\mathrm{MeV}$ electron. The $20 \mathrm{mg} \mathrm{cm}^{-2}$ in the Al tip hardly affects the energy loss of a $1-\mathrm{MeV}$ electron and similarly in a

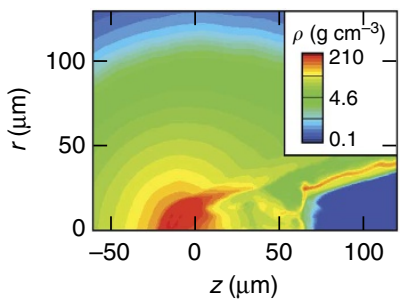

Figure 8 | Density map and electron stopping. (a) Simulated massdensity distribution of the improved implosion at the time of shock breakout at $3.96 \mathrm{~ns}$, which provides an areal density of $500 \mathrm{mg} \mathrm{cm}^{-2}$. (b) Calculated range of fast electrons in compressed deuterated plastic and DT.

the $15-\mu$ m-thick Au tip. On the other hand, the angular scattering increases with the atomic number of the material and the mean free path for $90^{\circ}$ scattering is much shorter in $\mathrm{Au}\left(46 \mathrm{mg} \mathrm{cm}^{-2}\right)$ than in $\mathrm{Al}\left(151 \mathrm{mg} \mathrm{cm}^{-2}\right)^{30}$. As scattering increases the divergence of the electron beam when it passes through the tip, the amount of electrons that intercept the dense plasma (the electrons that are available for ignition) decreases. The Al tip might therefore have some advantage compared with the Au-tip design.

A simple expression for maximum $\rho R$ was derived in ref. 31 for an imploded DT capsule filled with $1 \mathrm{~atm}$ of DT gas, which makes it possible for the maximum $\rho R$ to be scaled with the drive laser energy $E_{\mathrm{L}}$, the laser wavelength $\lambda$ and the adiabat $\alpha_{\mathrm{inn}}$ of the inner portion of the shell $(\rho R)_{\max }\left(\mathrm{mg} \mathrm{cm}^{-2}\right) \approx 260 E_{\mathrm{L}}^{1 / 3}(\mathrm{~kJ})$ $[0.35 / \lambda(\mu \mathrm{m})]^{0.25} / \alpha_{\mathrm{inn}}^{0.54}$. For the current experiment, $E_{\mathrm{L}}=18 \mathrm{~kJ}$, $\lambda=0.35 \mu \mathrm{m}$ and $\alpha_{\mathrm{inn}}=3.4$, for which the simple expression yields $\sim 350 \mathrm{mg} \mathrm{cm}^{-2}$, only $\sim 17 \%$ higher than the actual value, showing that the scaling model can be used to estimate the expected $(\rho R)_{\max }$ for different energies and adiabats. For a laser energy of $1.9 \mathrm{MJ}$, which was used in a recent experiment ${ }^{6}$ at the National Ignition Facility, and for $\alpha_{\text {inn }}=3.4$, a value of $(\rho R)_{\max }=1,600 \mathrm{mg} \mathrm{cm}^{-2}$ is estimated, which is a factor of $\sim 4.6$ higher than with OMEGA. A higher areal density allows for particle stopping up to a higher kinetic energy and, given a certain distribution of kinetic energies, relaxes the requirement to ignite the fuel.

Our simulations indicate that an even higher $\rho R$ might be achievable on OMEGA by removing the air from the shell and reducing the power of the laser pulse picket. Figure 8 a shows the calculated mass-density map at shock breakout for the improved design. The mass and energy of the hot spot are decreased, which delays the breakout of the cone tip by reducing the pressure on the cone tip. Fuel stagnation is closer to the target centre with a higher density and higher areal density. It brings the shock breakout as close as $80 \mathrm{ps}$ to the peak compression time. The ablation velocity is only $8 \%$ less for the reduced-power picket implosion. In general, a larger ablation velocity improves the 
hydrodynamic stability ${ }^{15}$, but those implosions use thick shells and a low implosion velocity so the slight reduction in ablation velocity does not significantly affect hydrodynamic stability. The main effect of the reduced picket is a lower $\alpha_{\text {inn }}$ and reduced mass and temperature in the hot spot. At the time of shock breakout, the areal density reaches $500 \mathrm{mg} \mathrm{cm}^{-2}, 83 \%$ of $\rho R_{\max }=600 \mathrm{mg} \mathrm{cm}^{-2}$. It is important to note that such high areal densities provide sufficiently dense plasma to stop fast electrons. Figure $8 \mathrm{~b}$ shows the calculated average range of fast electrons in compressed deuterated plastic (solid curve) including blooming and straggling ${ }^{30}$. Electrons up to $1.8 \mathrm{MeV}$ will completely range out in a $\rho R=500-\mathrm{mg} \mathrm{cm}^{-2}$ plastic plasma, whereas a $200-\mathrm{mg} \mathrm{cm}^{-2}$ plasma stops electrons up to $0.9 \mathrm{MeV}$. The areal density across the dense core is about twice that value $(2 \rho R)$ for electrons propagating from the centre of the cone tip to the centre of the dense core. Therefore, electrons of up to $1.5 \mathrm{MeV}$ can be stopped along this trajectory for the present implosion and up to $3 \mathrm{MeV}$ for the optimized implosion. The dashed curve in Fig. 8b represents the fast-electron range in compressed DT, which is about twice that of plastic. DT plasma with $550 \mathrm{mg} \mathrm{cm}^{-2}$ will stop a $1-\mathrm{MeV}$ electron. Similar areal densities are expected for imploded cryogenic DT shells on OMEGA. In fact, $\rho R=300 \mathrm{mg} \mathrm{cm}^{-2}$ has been measured on OMEGA in spherical cryogenic DT implosions (without a cone $)^{32}$. National Ignition Facility implosions with cryogenic DT compressed to $1,600 \mathrm{mg} \mathrm{cm}^{-2}$ will stop electrons up to $2.4 \mathrm{MeV}$ propagating from outside to the plasma centre and up to 4.5-MeV electrons across the dense core.

Injecting a short-pulse laser into the hollow cone produces fast electrons with energies of up to several $\mathrm{MeV}^{12}$. The coupling efficiency of the electron energy into the dense core depends on a number of parameters, including the standoff distance between the location where the fast electrons are produced and the dense plasma, the divergence and energy distribution of the electrons, the transport dynamics through a potential pre-plasma inside the cone $^{12}$ and through the cone wall, and the areal density of the compressed plasma. There is an ongoing effort to quantify this coupling efficiency in OMEGA experiments by characterizing the spatial distribution of fast-electron energy in the compressed core using $\mathrm{Cu}$-doped plastic shells and imaging the fast-electronexcited $\mathrm{Cu} K_{\alpha}$ fluorescence emission ${ }^{33,34}$.

\section{Methods \\ Cone-in-shell target. A hollow gold cone with an inner full opening angle of $34^{\circ} \pm 1^{\circ}$, a sidewall thickness of $15 \mu \mathrm{m}$ and a small inner $40-\mu \mathrm{m}$ circular flat tip was inserted into a $42-\mu \mathrm{m}$-thick plastic shell with a $14-\mu \mathrm{m}$ outer $\mathrm{CH}$ layer, a $28-\mu \mathrm{m}$ inner strong deuterated plastic layer and an outer diameter of $\sim 870 \mu \mathrm{m}$. The shell was filled with $\sim 0.8$ atm of air at shot time. A thin $(\sim 2-\mu \mathrm{m})$ gold layer inside the cone end served as a mounting layer for the Al tip. The Al tip was $60 \mu \mathrm{m}$ thick on axis and $78 \mu \mathrm{m}$ in diameter.}

Backlighting of undriven target. Figure 3a shows a backlighter image of an undriven target. The Al tip was well centred on the Au cone within $\pm 5 \mu \mathrm{m}$. The image was corrected for spatially varying backlighter intensity, similar to that described in ref. 18, and for hard X-rays that were generated in the foil and scattered in the diagnostic. The expected $8-\mathrm{keV}$ X-ray transmission through the Au cone wall is $\sim 7 \times 10^{-6}$, and the area shadowed by the cone should be free of $\mathrm{X}$-ray signal. The high-energy X-ray background that appeared in this area was therefore subtracted from the measured image before the $2 \mathrm{D}$ backlighter intensity distribution was reconstructed. The image was then normalized to the backlighter intensity distribution, resulting in the image shown in Fig. 3a. A vertical lineout through the centre of the cylindrical Al tip is well fitted by a convolution of the radial transmission function through a disk, $\exp \left[-2 \kappa \sqrt{r^{2}-y^{2}}\right]$, with a Gaussian curve, representing the spatial resolution of the imaging system (see Fig. 3b). Here $\kappa$ is the absorption coefficient, $r$ is the disk radius and $y$ is the coordinate of observation. The radius of the $\mathrm{Al}$ tip was $r=39 \mu \mathrm{m}$ and the absorption coefficient of cold aluminum at $8.048 \mathrm{keV}$ was $\kappa=1.265 \times 10^{-2} \mu \mathrm{m}^{-1}$. The fit yielded an independent measurement of the magnification of $14.8 \pm 0.1$, which is in excellent agreement with the magnification of 14.7 calculated from engineering drawings. A spatial resolution of $22 \pm 3 \mu \mathrm{m}$ was inferred from the fit, which is a factor of
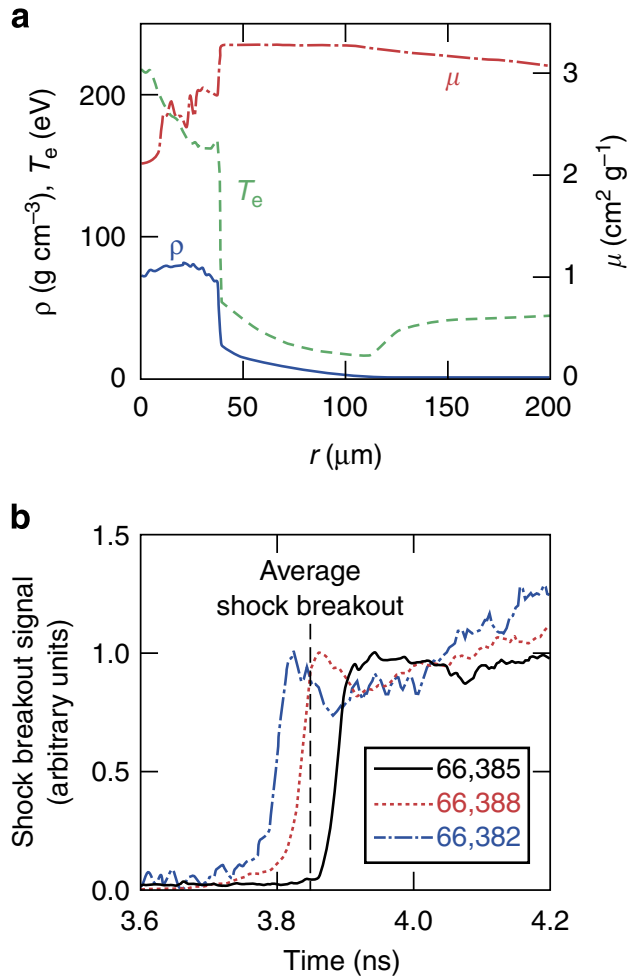

Figure 9 | Mass-absorption coefficient and shock breakout. (a) NLTE calculation of the radial profile of the mass-absorption coefficient ( $\mu$; dashed-dotted curve) of a $C_{\mathbf{1}} D_{\mathbf{1 . 4}}$ plasma at a photon energy of $8.048 \mathrm{keV}$ along the photon path through the dense plasma at peak compression (4.05 ns) using density (solid) and temperature (dashed) profiles from the radiation hydrodynamic simulation. (b) Shock breakout measured with a streaked optical pyrometer for three different implosions. The average shock breakout time is marked by the vertical dashed line.

$\sim 2$ worse than the resolution of $\sim 10 \mu \mathrm{m}$ reported in ref. 24 . The reason is that the resolution tests in ref. 24 were done with an aperture in front of the crystal, which mitigates the astigmatism of the spherical crystal and improves the spatial resolution, whereas in the current experiment no aperture was used to maximize the signal.

Absorption coefficient and mass-absorption coefficient. The optical depth $\tau$ is given by the integral over the absorption coefficient $\kappa$ along the path of an X-ray photon through the dense plasma $\tau=\int \kappa(x) \mathrm{d} x$ measured at the specific backlighter photon energy. The measured optical-depth profiles $\tau(y)$ were Abel inverted to infer the absorption coefficient

$$
\kappa(r)=-\frac{1}{\pi} \int_{r}^{\infty}\left(\frac{\mathrm{d}}{\mathrm{d} y} \tau(y)\right) \frac{\mathrm{d} y}{\sqrt{y^{2}-r^{2}}}
$$

using an iterative Abel algorithm ${ }^{35}$. The assumption of a straight-line photon path is justified at high photon energies. The absorption coefficient is equal to the product of the mass-absorption coefficient $\mu$ and the mass density $\rho$, where $\mu$ is a function of plasma electron temperature $T_{\mathrm{e}}$ and $\rho$. A steady-state nonlocal thermal electron calculation of the mass-absorption coefficient $\mu$ was performed with the SPECT $3 D$ software ${ }^{26}$ for $C_{1} D_{1.4}$ plasma at a photon energy of $8.048 \mathrm{keV}$ and the density profile was inferred $(\rho=\kappa / \mu)$. In the range of densities and temperatures of interest here, $\mu$ varies between $\sim 3.3$ and $\sim 2 \mathrm{~cm}^{2} \mathrm{~g}^{-1}$ along the path of integration, as shown in Fig. 9a. Profiles of $\rho$ and $T_{\mathrm{e}}$ from DRACO simulations along the same $y$ direction were used to calculate $\mu$ versus position. The technique is sensitive to the assumed temperature and density. A lower temperature results in a higher $\mu$ and therefore in a lower inferred $\rho$. The sensitivity of $\rho$ on the temperature was studied by multiplying the temperature profile from the hydrodynamic simulation by 0.9 and 1.1 and repeating the data analysis. The resulting inferred curves $\rho(r)$ are very similar $(< \pm 3 \%$ deviation) for $r>20 \mu \mathrm{m}$ and deviate slightly more $(< \pm 12 \%$ deviation) at the centre of the compressed target. The sensitivity of $\mu$ on the density is less important and is minimized by an iterative procedure. The inferred density profile is reiterated and $\mu$ is recalculated. The process quickly converges. 
Shock breakout measurements. The abrupt onset of optical emission was measured temporally and spatially resolved from the inside of the cone with a streaked optical pyrometer (SOP) ${ }^{36}$ in the $\sim 600$ - to $750-\mathrm{nm}$-wavelength range and with two velocity interferometer systems for any reflector (VISARs) ${ }^{37}$ at a $532-\mathrm{nm}$ wavelength. Figure $9 \mathrm{~b}$ shows the SOP signal for three different implosions. Slight differences in pulse shape and shell thickness give rise to the variation in breakout time. The SOP integrates over a time period of $150 \mathrm{ps}$, determined by the slit width and the sweep speed of the streak camera. The signal is differentiated with respect to time and the first peak determines shock breakout with an accuracy of \pm 40 ps. The VISAR achieves a higher accuracy of \pm 20 ps in measuring shock breakout with the help of a faster streak camera. For each shot, the measurements of all three instruments are in agreement within the experimental error.

\section{References}

1. Boehly, T. R. et al. Initial performance results of the OMEGA laser system. Opt. Commun. 133, 495-506 (1997).

2. Moses, E. I., Boyd, R. N., Remington, B. A., Keane, C. J. \& Al-Ayat, R. The National Ignition Facility: ushering in a new age for high energy density science. Phys. Plasmas 16, 041006 (2009).

3. Nuckolls, J., Wood, L., Thiessen, A. \& Zimmerman, G. Laser compression of matter to super-high densities: thermonuclear (CTR) applications. Nature 239, 139-142 (1972).

4. Drake, R. P. Shock Wave and High Pressure Phenomena (Springer, Berlin, 2006).

5. Remington, B. A., Drake, R. P. \& Ryutov, D. D. Experimental astrophysics with high power lasers and $Z$ pinches. Rev. Mod. Phys. 78, 755-807 (2006).

6. Hurricane, O. A. et al. Fuel gain exceeding unity in an inertially confined fusion implosion. Nature 506, 343-348 (2014).

7. Tabak, M. et al. Ignition and high gain with ultrapowerful lasers. Phys. Plasmas 1, 1626-1634 (1994).

8. Betti, R. et al. Shock ignition of thermonuclear fuel with high areal density. Phys. Rev. Lett. 98, 155001 (2007).

9. The state of the art of both concepts are reviewed in a series of articles that recently appeared in the journal of Nucl. Fusion 54, Number 5, Special issue: Collection of Special Topic papers on inertial confinement fusion (2014).

10. Kodama, R. et al. Fast heating of ultrahigh-density plasma as a step towards laser fusion ignition. Nature 412, 798-802 (2001).

11. Kodama, R. et al. Nuclear fusion: Fast heating scalable to laser fusion ignition. Nature 418, 933-934 (2002).

12. Theobald, W. et al. Initial cone-in-shell fast-ignition experiments on OMEGA. Phys. Plasmas 18, 056305 (2011).

13. Arikawa, Y. et al. A experimental study on the energy coupling efficiency from the heating laser to core plasma in the fast ignition experiment. Bull. Am. Phys. Soc 58, 372 (2013).

14. Atzeni, S. \& Meyer-ter-Vehn, J. International Series of Monographs on Physics (Clarendon Press, 2004).

15. Goncharov, V. N. et al. Improved performance of direct-drive inertial confinement fusion target designs with adiabat shaping using an intensity picket. Phys. Plasmas 10, 1906-1918 (2003).

16. Zhou, C. D. et al. High- $\rho$ R implosions for fast-ignition fuel assembly. Phys. Rev. Lett. 98, 025004 (2007).

17. Landen, O. L. et al. X-ray backlighting for the National Ignition Facility (invited). Rev. Sci. Instrum 72, 627-634 (2001).

18. Marshall, F. J. et al. Plasma-density determination from X-ray radiography of laser-driven spherical implosions. Phys. Rev. Lett. 102, 185004 (2009).

19. Stephens, R. B., Hatchett, S. P., Turner, R. E., Tanaka, K. A. \& Kodama, R. Implosion of indirectly driven reentrant-cone shell target. Phys. Rev. Lett. 91, 185001 (2003)

20. Stephens, R. B. et al. Implosion hydrodynamics of fast ignition targets. Phys. Plasmas 12, 056312 (2005).

21. Stoeckl, C. et al. Direct-drive fuel-assembly experiments with gas-filled, cone-in-shell, fast-ignitor targets on the OMEGA laser. Plasma Phys. Control. Fusion 47, B859-B867 (2005)

22. Waxer, L. J. et al. High-energy petawatt capability for the OMEGA laser. Opt. Photonics News 16, 30-36 (2005).

23. Nilson, P. M. et al. Time-resolved measurements of hot-electron equilibration dynamics in high-intensity laser interactions with thin-foil solid targets. Phys. Rev. Lett. 108, 085002 (2012).

24. Stoeckl, C. et al. A spherical crystal imager for OMEGA EP. Rev. Sci. Instrum 83, 033107 (2012).
25. Radha, P. B. et al. Multidimensional analysis of direct-drive, plastic-shell implosions on OMEGA. Phys. Plasmas 12, 056307 (2005).

26. MacFarlane, J. J., Golovkin, I. E., Wang, P., Woodruff, P. R. \& Pereyra, N. A. SPECT3D-a multi-dimensional collisional-radiative code for generating diagnostic signatures based on hydrodynamics and PIC simulation output. High Energy Density Phys 3, 181-190 (2007).

27. Goncharov, V. N. et al. Performance of direct-drive cryogenic targets on OMEGA. Phys. Plasmas 15, 056310 (2008).

28. Froula, D. H. et al. Increasing hydrodynamic efficiency by reducing cross-beam energy transfer in direct-drive implosion experiments. Phys. Rev. Lett. 108, 125003 (2012).

29. Wyckoff, H. O. Stopping powers for electrons and positions. ICRU Report No. 37 (International Commission on Radiation Units and Measurements, Inc., 1984).

30. Solodov, A. A. \& Betti, R. Stopping power and range of energetic electrons in dense plasmas of fast-ignition fusion targets. Phys. Plasmas 15, 042707 (2008).

31. Zhou, C. \& Betti, R. Hydrodynamic relations for direct-drive fast-ignition and conventional inertial confinement fusion implosions. Phys. Plasmas 14, 072703 (2007).

32. Goncharov, V. N. et al. Demonstration of the highest deuterium-tritium areal density using multiple-picket cryogenic designs on OMEGA. Phys. Rev. Lett. 104, 165001 (2010).

33. Wei, M. S. et al. Study of fast electron transport into imploded high-density plasmas using Cu-doped CD shell targets. Bull. Am. Phys. Soc. 56, 146 (2011)

34. Jarrott, L. C. Fast electron transport and spatial energy deposition in imploded fast ignition cone-in-shell targets. in 56th Annual Meeting of the APS Division of Plasma Physics New Orleans, (2014).

35. Vicharelli, P. A. \& Lapatovich, W. P. Iterative method for computing the inverse Abel transform. Appl. Phys. Lett. 50, 557-559 (1987).

36. Miller, J. E. et al. Streaked optical pyrometer system for laser-driven shock-wave experiments on OMEGA. Rev. Sci. Instrum. 78, 034903 (2007).

37. Celliers, P. M. et al. Line-imaging velocimeter for shock diagnostics at the OMEGA Laser Facility. Rev. Sci. Instrum. 75, 4916-4929 (2004).

38. Lerche, R. A., Phillion, D. W. \& Tietbohl, G. L. 25 ps neutron detector for measuring ICF-target burn history. Rev. Sci. Instrum. 66, 933-935 (1995).

\section{Acknowledgements}

This material is based upon work supported by the Department of Energy National Nuclear Security Administration under Award Number DE-NA0001944, the OFES Fusion Science Center grant No. DE-FC02-04ER54789, the OFES ACE Fast Ignition grant No. DE-FG02-05ER54839, the DOE Laboratory Basic Science Program, the University of Rochester, and the New York State Energy Research and Development Authority. The support of DOE does not constitute an endorsement by DOE of the views expressed in this article. We thank R. Betti for discussions. J.J.S. participated in this work thanks to funding from the French National Agency for Research (ANR) and the competitiveness cluster Alpha-Route des Lasers through project TERRE ANR-2011BS04-014.

\section{Author contributions}

W.T. designed and executed the experiment as principle investigator with help from C.S F.N.B., V.Yu.G., H.H., L.C.J., F.J.M., C.M., P.M.N., J.J.S., H.S., P.K.P., F.P. and M.S.W.; C.S., C.M. and G.F. developed the crystal imager. Data analysis was performed by G.M., S.I., F.J.M. and W.T. All simulations and calculations were performed by A.A.S with help from R.E., A.S. and K.S.A. Targets were manufactured by E.M.G., M.S.W. and R.B.S W.T. lead the writing of the manuscript and A.A.S, H.S.M., C.S., F.N.B., T.C.S., R.B.S. and M.S.W. helped in preparation of the text. Figures were prepared by W.T. and A.A.S

\section{Additional information}

Competing financial interests: The authors declare no competing financial interests.

Reprints and permission information is available online at http://npg.nature.com/ reprintsandpermissions/

How to cite this article: Theobald, W. et al. Time-resolved compression of a capsule with a cone to high density for fast-ignition laser fusion. Nat. Commun. 5:5785 doi: $10.1038 /$ ncomms6785 (2014). 